\title{
Effect of latrogenic Traction during Macular Peeling Surgery on Postoperative Microperimetry
}

\author{
Christoph Leisser Stefan Palkovits Julius Hienert Marlies Ullrich \\ Hannah Zwickl Stefan Georgiev Oliver Findl \\ VIROS - Vienna Institute for Research in Ocular Surgery, A Karl Landsteiner Institute, Hanusch Hospital, \\ Vienna, Austria
}

\section{Keywords}

Epiretinal membranes · Membrane peeling $\cdot$ Intraoperative Optical Coherence Tomography · Microperimetry

\begin{abstract}
Introduction: Vitrectomy with peeling of epiretinal membrane (ERM) and internal limiting membrane offers the chance for improvement of metamorphopsia and visual acuity. Microscope integrated intraoperative optical coherence tomography (iOCT) enables real-time imaging of retinal alterations during peeling, such as intraoperative transient retinal thickening owing to tractional forces during peeling. The aim of our study was to measure the amounts of transient retinal thickening due to tractional forces during membrane peeling, as documented with IOCT, and to analyze possible effects on postoperative retinal function. Methods: This prospective, monocenter study included patients scheduled for pars plana vitrectomy with membrane peeling due to an idiopathic ERM. During peeling, an IOCT device (ReScan700, Carl Zeiss Meditec AG) with continuous OCT-assistance during the peeling procedure, and video documentation of the peeling procedure, was used for the assessment of intraoperative transient retinal thickening owing to trac-
\end{abstract}

tional forces during peeling. Directly before and 3 months after surgery, macular-OCT scans and microperimetry were performed. Results: Twenty-five eyes of 25 patients were included in the study. Microperimetry could be performed in all patients, while iOCT documentation could be analyzed in 22 patients. Transient retinal thickening owing to tractional forces during peeling could be observed in 14 patients (64\%), with a median thickening to $143 \%$ of the normal (preoperative) retinal thickness at that location (IQR 132-163). Six patients (24\%) developed new deep microscotomata as seen in microperimetry 3 months after surgery, among them were 2 patients who also had transient retinal thickening during peeling. Conclusion: New deep microscotomata developed only in a minority of patients with transient retinal thickening owing to tractional forces during peeling.

(c) 2020 S. Karger AG, Basel

\section{Introduction}

Epiretinal membranes (ERM), a macular disorder hypothesized to be developed from hyalocytes and Müller cells $[1,2]$, lead to metamorphopsia and a decrease in visual acuity. Hyalocytes and Müller cells have been found karger@karger.com

www.karger.com/ore

Karger"
(C) 2020 S. Karger AG, Basel

Hanusch Hospital

Heinrich Collin Strasse 30

AT-1140 Vienna (Austria)

oliver@findl.at 
in epimacular tissue [1] and seem to play an important role in ERM formation and contraction [2]. Vitrectomy with peeling of ERM, first reported in 1978 [3], offers the chance for improvement of metamorphopsia and visual acuity. Combined ERM peeling and peeling of the internal limiting membrane (ILM) resulted in significantly lower recurrence rates of ERM [4-7], but results concerning the visual acuity outcomes are controversial. While one meta-analysis reported improved visual acuity in the combined ERM and ILM peeling group compared to the ERM peeling group [4], other meta-analyses and reviews found no benefit of additional ILM peeling on visual acuity [5-8]. Since microscotomata after peeling of the ILM were reported [9-11], there has been a discussion of whether ERM peeling combined with or without ILM peeling should be performed in routine cases.

Adherence of ERM to the retinal surface is different from case to case, resulting in different amounts of tractional force needed for successful peeling. Microscope integrated intraoperative optical coherence tomography (iOCT) [12-14] offers real-time imaging of retinal alterations during peeling, such as subfoveal and extrafoveal hyporeflective zones $[15,16]$, and helps in guiding the peeling procedure with respect to the need for ERM staining with chromovitrectomy dyes and visualization of optimal starting points $[17,18]$. The aim of our study was to measure the amounts of transient retinal thickening due to tractional forces during membrane peeling, as documented with iOCT, and to analyze possible effects on postoperative retinal function, as assessed by microperimetry.

\section{Methods}

This prospective, monocenter study included patients scheduled for pars plana vitrectomy with membrane peeling due to an idiopathic ERM at the ophthalmic department of the Hanusch Hospital in Vienna, Austria, between September 2015 and June 2018. The inclusion criteria were an age above 21 years and the presence of idiopathic ERM with indication for surgery, defined by loss in visual acuity (assessed by clinical judgement) and/or significant metamorphopsia. The exclusion criteria were a macular edema due to any other reason than ERM (diagnosed with preoperative SD-OCT and fundoscopy). Research and measurements followed the tenets of the Declaration of Helsinki and were approved by the local ethics committee of the city of Vienna. Written informed consent was obtained from all patients in the study.

All patients underwent 23-G 3-port pars plana vitrectomy with ERM peeling as a first step and, in cases with persistence of wrinkling of the retinal surface, peeling of the ILM was performed as a second step. Two experienced vitreoretinal surgeons (O.F., C.L.) participated in the study. Staining of the membranes was performed with a trypan blue and brilliant blue G-based dye (Mem-
branBlue Dual, Dutch Ophthalmic Research Center, Zuidland, The Netherlands) and peeling was performed using an end-gripping forceps, with a tano-scraper or a micropic used when necessary to open the membranes, typically in a location temporal to the macula. During peeling, an iOCT device (Rescan 700, Carl Zeiss Meditec AG, Jena, Germany) with continuous OCT assistance during the peeling procedure and video documentation of the peeling procedure was used.

Directly before and 3 months after surgery, macular-OCT scans with a technically comparable stand-alone SD-OCT (CirrusOCT, Carl Zeiss Meditec AG) and microperimetry (MP3, Nidek, Japan) were performed. Retinal thickness was measured from iOCT images, exported from the continuous video documentation during surgery, with Image (NIH, Bethesda, MD, USA) and alterations in retinal thickness due to iatrogenic traction of tissue during peeling was expressed in the percent of thickness increase compared to the preoperative retinal thickness measured at that specific location. The preoperative retinal thickness at that specific location was obtained from the iOCT images at the time point directly before membrane peeling and retinal thickness values were compared to those during membrane peeling for documentation of the amount of retinal thickening during membrane peeling. The amount of maximal thickening at a specific location was measured from iOCT images. Furthermore, it was analyzed from the continuous iOCT video documentation whether retinal thickening during membrane peeling was transient (in case the retinal thickness was normal again after the release of peeling-induced traction) or persistent. Areas of retinal thickening (transient or persistent) were localized in microperimetric outcomes according to landmarks, such as retinal vessels, for possible development of new deep microscotomata. We defined deep microscotomata as retinal points with a retinal sensitivity below $5 \mathrm{~dB}$. Furthermore, the occurrence of subfoveal or extrafoveal hyporeflective zones during membrane peeling was assessed during review of the continuous iOCT video documentation. Subfoveal or extrafoveal hyporeflective zones are a tent-shaped neurosensory elevation of the retina $[15,16]$, resulting from traction during membrane peeling, typically at areas of transient or persistent retinal thickening during membrane peeling, that usually disappear immediately after peeling-induced traction is released $[15,16]$.

Microperimetry was performed, as already described in a previous study with patients with macular holes [19], in a dark room with the contralateral eye patched during measurements. Measurement points were tested with a special algorithm designed for testing macular points. The device MP3 (Nidek, Tokyo, Japan) has integrated fundus imaging with a $45^{\circ}$ field of view and resolution of $12 \mathrm{mpx}$ and motion tracking to ensure the precise stimulation of certain locations of the retina. Microperimetry was performed with a tracking rate for motion tracking of $30 \mathrm{~Hz}$ and the range of stimulus luminance for retinal sensitivity testing was $0-34 \mathrm{~dB}$ with a maximum stimulation luminescence of 10,000 asb and a background illumination of 34.1 asb. In case of significant eye movements or when patients needed a break, the test was paused.

In cases of coexisting vision affecting cataract, combined vitrectomy with membrane peeling and phacoemulsification with implantation of an intraocular lens was performed. Use of air-tamponade at the end of surgery was used at the surgeon's discretion. Changes in pre- and postoperative retinal thickness in micrometers were assessed by SD-OCT using the central subfield thickness 

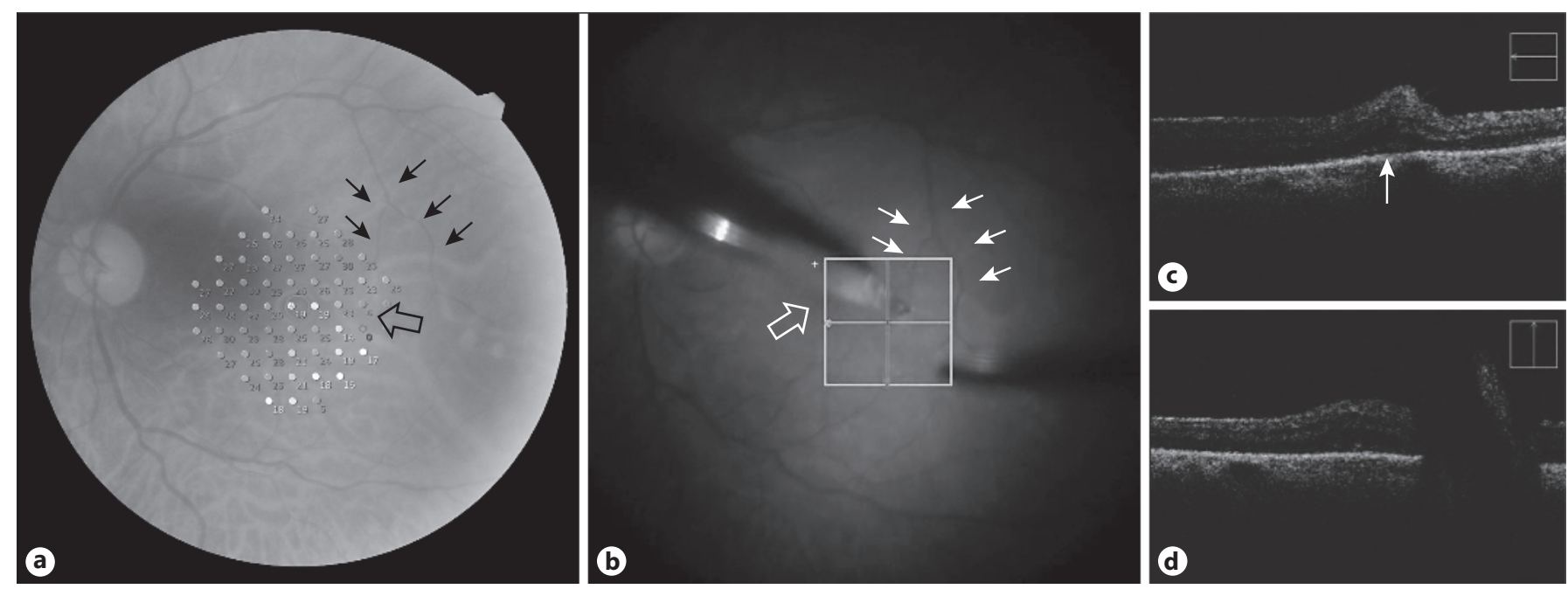

Fig. 1. a New postoperative deep microscotomata in microperimetry 3 months after surgery can be detected. One of these microscotomata (large arrow) lies on the corresponding area of transient retinal thickening due to tractional forces during membrane peeling. For exact localization of corresponding areas, we used landmarks. b The small arrows depict retinal vessels and the large arrow the fovea, both used as landmarks for exactly localizing the area of transient retinal thickening due to tractional forces during peeling in the microperimetry outcomes. As the fundus image, obtained from the continuous iOCT documentation during membrane peeling, passed through the operating microscope and is therefore inverted according to the sitting position of the surgeon, we reinverted it for better localization of the landmarks. c, d In the iOCT transient retinal thickening due to tractional forces during membrane peeling can be shown with a discrete extrafoveal hyporeflective zone (arrow). of the macular thickness analysis with a diameter of $1 \mathrm{~mm}$ including the fovea.

The main outcome measures of the study were the amount of retinal thickening during peeling in percent of the normal retinal thickness at that specific location, and the occurrence of new postoperative deep microscotomata in microperimetry after membrane peeling. Secondary outcome measures were distance-corrected visual acuity (DCVA), and occurrence of subfoveal or extrafoveal hyporeflective zones.

Statistical analysis was performed in a descriptive fashion for mean values, standard deviation, median and range with Excel 2016 (Microsoft, Redmond, WA, USA). In case of a gaussian distribution (tested with the Shapiro-Wilk test), mean values and standard deviation were calculated and the $t$ test was applied. Otherwise the median, IQR, and range were calculated, and the MannWhitney $U$ test, or Wilcoxon matched-pairs test in the case of paired data, were applied. The Shapiro-Wilk test, IQR, $t$ test, Wilcoxon-Mann-Whitney U test, and Wilcoxon matched-pairs test were performed using the software tool BiAS (epsilon-Verlag, Frankfurt, Germany). $p<0.05$ was regarded to indicate significant differences between groups.

\section{Results}

Twenty-five eyes of 25 patients with idiopathic ERM scheduled for 23-G pars plana vitrectomy with membrane peeling were included into this study. The mean age of the patients was 72.4 years (SD 4.5, range 64-82); 14 were right eyes and 11 were left eyes, and 13 subjects were females and 12 males. Phacovitrectomy was performed in 11 patients due to coexisting vision-affecting cataract. Twelve patients underwent additional ILM peeling and 16 patients had air-tamponade at the end of surgery. One eye underwent gas-tamponade and endolaser due to peripheral retinal detachment detected during surgery.

\section{Intraoperative Retinal Thickening Observed by iOCT}

Among the total of 25 patients included, iOCT could be analyzed in 22, while in 3 patients the documentation of the iOCT during peeling was not possible due to technical problems with the device. Transient retinal thickening owing to tractional forces during peeling could be observed in 14 patients (64\%), with a median thickening to $143 \%$ of the normal (preoperative) retinal thickness at that location (IQR 132-163). In all patients transient retinal thickening lasted until peeling was successfully performed at that specific location, and after the release of peeling-induced traction on retinal tissue, normal retinal thickness was restored again. Transient retinal thickening owing to tractional forces during peeling resulted in a transient extrafoveal hyporeflective zone in 1 patient (4\%; Fig. 1).
Effect of Iatrogenic Traction during Peeling on Postoperative Microperimetry
Ophthalmic Res 2021;64:273-279 DOI: $10.1159 / 000507633$ 

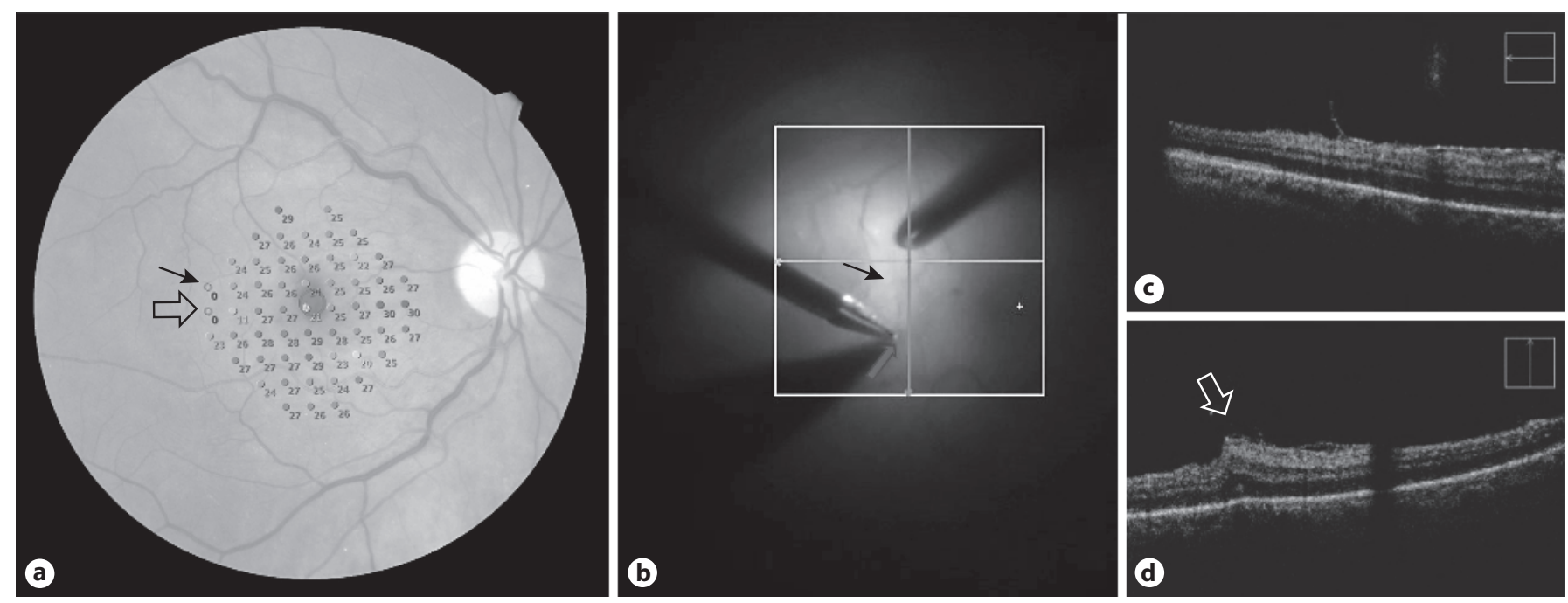

Fig. 2. a New postoperative deep microscotomata in the starting point area of membrane peeling (small arrows) in an eye with transient retinal thickening (large arrow) due to tractional forces during membrane peeling. b-d iOCT.

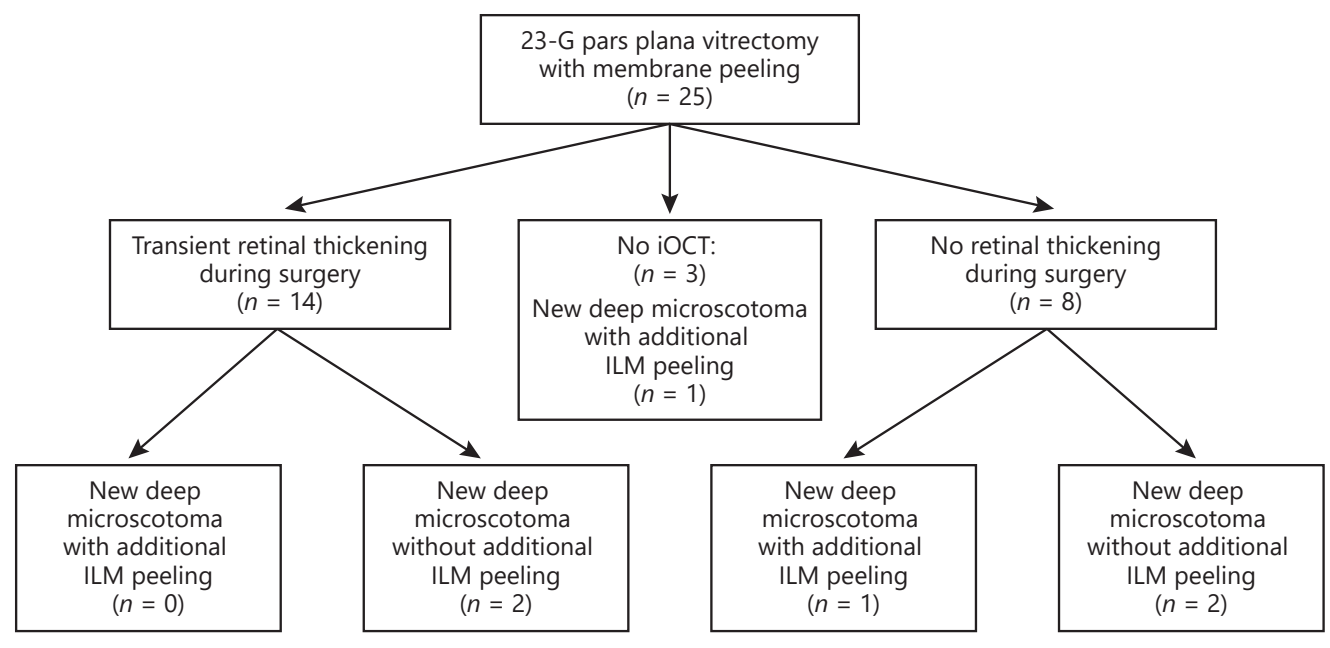

Fig. 3. An overview of the distribution of patients with transient retinal thickening during membrane peeling, corresponding new postoperative deep microscotoma, and additional ILM peeling.

\section{Occurrence of Deep Microscotomata in}

\section{Microperimetry}

Six patients (24\%) had new postoperative deep microscotoma in microperimetry 3 months after surgery, 2 in the temporal-inferior extrafoveal region and 4 in the temporal extrafoveal region. Corresponding retinal thinning in postoperative OCT at the location of these microscotoma was present in 4 (16\%) of these patients.
Among patients with transient retinal thickening owing to tractional forces during peeling, corresponding postoperative deep microscotoma could be observed in 2 patients ( 1 of these had a transient extrafoveal hyporeflective zone during surgery and postoperative retinal thinning in that area; Fig. 1). In another 3 patients, focal postoperative reductions of retinal sensitivity $(-3 \mathrm{~dB},-4 \mathrm{~dB}$ and $-9 \mathrm{~dB}$ ) occurred. 

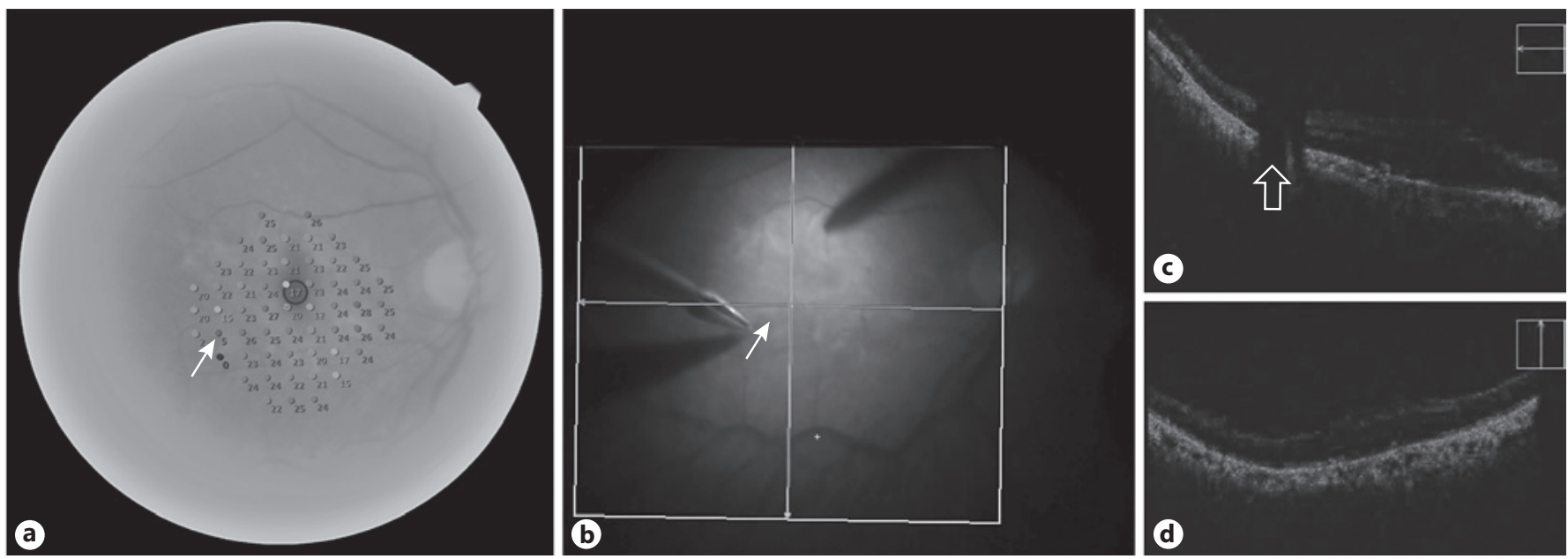

Fig. 4. a, b New postoperative deep microscotoma without transient retinal thickening in the iOCT (small arrows). c, d In the iOCT (right) a forceps-related artefact (large arrow) can be seen.

While 2 patients with new postoperative deep microscotomata, as mentioned above, both without additional ILM peeling, had transient retinal thickening owing to tractional forces during peeling in the iOCT (Fig. 1, 2), 3 patients with new postoperative deep microscotomata had no signs of transient retinal thickening owing to tractional forces during peeling in the $\mathrm{iOCT}$ at the areas of microscotomata. Among these patients, 1 patient underwent additional ILM peeling. Furthermore, in another patient with additional ILM peeling, a new postoperative deep microscotoma could be diagnosed. Unfortunately, in that patient the iOCT did not work appropriately during the procedure. An overview is provided in Figure 3. Neither occurrence of transient retinal thickening owing to tractional forces during peeling resulted in a significant different amount of development of postoperative deep microscotomata ( $p=0.6$; Fisher's exact test), nor additional ILM peeling $(p=0.7$; Fisher's exact test).

\section{Visual Acuity}

The median improvement of DCVA in eyes with transient retinal thickening owing to tractional forces during peeling (median +1.5 lines, IQR $0-3.25$, 95\% CI $0-4$ ) was not statistically different to eyes without (median +2.5 lines, IQR 2-3.75, 95\% CI 1-5; $p=0.17$ ). Patients who were phakic and pseudophakic at the follow-up 3 months after surgery were equally distributed between both subgroups. DCVA improved in $80 \%(n=20)$ and was equal to that prior to surgery in $20 \%(n=5)$.

Effect of Iatrogenic Traction during

Peeling on Postoperative Microperimetry

\section{Foveal Subfield Thickness}

The reduction of foveal subfield thickness in eyes with transient retinal thickening owing to tractional forces during peeling (median $-29 \mu \mathrm{m}, \mathrm{IQR}-91.75$ to $10,95 \%$ CI -97 to 10) was not statistically significant to eyes without (median $-88.5 \mu \mathrm{m}$, IQR -100.5 to $-53.5,95 \% \mathrm{CI}-131$ to $17 ; p=0.97)$.

\section{Discussion}

Transient retinal thickening owing to tractional forces during peeling could be observed in $64 \%$ of patients, with 1 of these patients having a corresponding extrafoveal hyporeflective zone during surgery. Furthermore, 6 patients (24\%) developed new deep microscotomata as seen in microperimetry 3 months after surgery, among them were 2 patients who also had transient retinal thickening during peeling.

Continuous iOCT documentation of membrane peeling, as described in our study, is a new application of iOCT. Surgeons may find it discomforting to actively move the scanning zone of the iOCT during peeling with the foot pedal to follow the area of membrane peeling, but up-to-date instrument-tracking tools for iOCT are not commercially available. Nevertheless, without continuous iOCT usage, real-time imaging of the retinal structures during membrane peeling is not possible and iatrogenic retinal pathologies, such as subfoveal and extrafoveal hyporeflective zones, tent-shaped neurosensory 
elevations of the retina, due to tractional forces during membrane peeling $[15,16]$, cannot be detected. The present study focused on retinal thickening due to tractional forces with or without subfoveal or extrafoveal hyporeflective zones. We described retinal thickening in percent of normal retinal thickness at specific locations to ensure interindividual comparability, as amounts of normal retinal thickness differ between patients.

Assuming mechanical traction during peeling as a cause for development of a microscotoma seems reasonable and our study outcomes show that transient retinal thickening owing to tractional forces during peeling can result in new deep microscotoma. Nevertheless, in the majority of patients iatrogenic traction during peeling did not result in loss of retinal sensitivity as assessed with microperimetry.

Since microscotomata after peeling of the ILM were reported [9-11], additional ILM peeling is controversially discussed. While several reports outline the reduction of ERM recurrence rates after ILM peeling [4-7], possible development of a higher number of microscotomata after ILM peeling [9-11] has to be considered during surgical planning. In our study, the majority of patients with additional ILM peeling did not develop new deep microscotomata and we were surprised to find 2 patients with new deep microscotomata, neither having had iatrogenic traction during peeling nor additional ILM peeling (Fig. 4).

\section{Conclusion}

New postoperative deep microscotomata developed only in a minority of patients with transient retinal thickening owing to tractional forces during peeling. Further- more, new deep microscotomata could also be observed in a minority of patients with or without additional ILM peeling without transient retinal thickening owing to tractional forces during peeling.

\section{Acknowledgements}

The authors would like to thank the whole team of the VIROS for their support of the study.

\section{Statement of Ethics}

All research and measurements followed the tenets of the Declaration of Helsinki and were approved by the local ethics committee of the city of Vienna. Written informed consent was obtained from all patients in the study.

\section{Disclosure Statement}

O. Findl is a scientific advisor for Carl Zeiss Meditec AG, but has no personal interest in the products mentioned. All authors declare that there are no conflicts of interest.

\section{Funding Sources}

No funding was required for this study.

\section{Author Contributions}

C.L.: conception of the study, data acquisition, statistical analysis, and preparation of the manuscript; S.P.: statistical analysis and critical review of the manuscript; J.H, M.U., H.Z., S.G.: data acquisition and critical review of the manuscript; O.F. conception of the study and critical review of the manuscript.

\section{References}

1 Zhao F, Gandorfer A, Haritoglou C, Scheler R, Schaumberger MM, Kampik A, et al. Epiretinal cell proliferation in macular pucker and vitreomacular traction syndrome: analysis of flat-mounted internal limiting membrane specimens. Retina. 2013 Jan;33(1):7788.

2 Kohno RI, Hata Y, Kawahara S, Kita T, Arita R, Mochizuki Y, et al. Possible contribution of hyalocytes to idiopathic epiretinal membrane formation and its contraction. Br J Ophthalmol. 2009 Aug;93(8):1020-6.

3 Machemer R. The surgical removal of epiretinal macular membranes (macular puckers).
Klin Monbl Augenheilkd. 1978 Jul;173(1): 36-42. German.

4 Chang WC, Lin C, Lee CH, Sung TL, Tung $\mathrm{TH}$, Liu JH. Vitrectomy with or without internal limiting membrane peeling for idiopathic epiretinal membrane: A meta-analysis. PLoS One. 2017 Jun;12(6):e0179105.

5 Schechet SA, DeVience E, Thompson JT. The effect of internal limiting membrane peeling on the idiopathic epiretinal membrane surgery, with a review of the literature. Retina. 2017 May;37(5):873-80.

6 Azuma K, Ueta T, Eguchi S, Aihara M. Effects of internal limiting membrane peeling com- bined with removal of idiopathic epiretinal membrane: a systematic review of literature and meta-analysis. Retina. 2017 Oct;37(10): 1813-9.

7 Díaz-Valverde A, Wu L. To peel or not to peel the internal limiting membrane in idiopathic epiretinal membranes. Retina. 2018 Sep; 38(Suppl 1):S5-11.

8 Liu H, Zuo S, Ding C, Dai X, Zhu X. Comparison of the effectiveness of pars plana vitrectomy with and without internal limiting membrane peeling for idiopathic retinal membrane removal: a meta-analysis. J Ophthalmol. 2015;2015:974568. 
9 Grimbert P, Lebreton O, Weber M. Optical coherence tomography and microperimetry after internal limiting membrane peeling for epiretinal membrane. J Fr Ophtalmol. 2014 Jun;37(6):434-41. French.

10 Ripandelli G, Scarinci F, Piaggi P, Guidi G, Pileri M, Cupo G, et al. Macular pucker: to peel or not to peel the internal limiting membrane? A microperimetric response. Retina. 2015 Mar;35(3):498-507.

11 Deltour JB, Grimbert P, Masse H, Lebreton O, Weber M. Detrimental effects of active internal limiting membrane peeling during epiretinal membrane surgery: microperimetric analysis. Retina. 2017 Mar;37(3): 544-52.

12 Tao YK, Ehlers JP, Toth CA, Izatt JA. Intraoperative spectral domain optical coherence tomography for vitreoretinal surgery. Opt Lett. 2010 Oct;35(20):3315-7.
13 Binder S, Falkner-Radler CI, Hauger C, Matz $\mathrm{H}$, Glittenberg C. Feasibility of intrasurgical spectral-domain optical coherence tomography. Retina. 2011 Jul-Aug;31(7):1332-6.

14 Ehlers JP, Tao YK, Farsiu S, Maldonado R, Izatt JA, Toth CA. Integration of a spectral domain optical coherence tomography system into a surgical microscope for intraoperative imaging. Invest Ophthalmol Vis Sci. 2011 May;52(6):3153-9.

15 Leisser C, Hackl C, Hirnschall N, Luft N, Döller B, Draschl P, et al. Visualizing macular structures during membrane peeling surgery with an intraoperative spectral-domain optical coherence tomography device. Ophthalmic Surg Lasers Imaging Retina. 2016 Apr;47(4):328-32.

16 Leisser C, Hackl C, Hirnschall N, Findl O. Effect of subfoveal and extrafoveal hyporeflective zones due to iatrogenic traction during membrane peeling for epiretinal membranes on postoperative outcomes. Ophthalmologica. 2019. doi: 10.1159/000505214.
17 Falkner-Radler CI, Glittenberg C, Gabriel M, Binder S. Intrasurgical microscope-integrated spectral domain optical coherence tomography-assisted membrane peeling. Retina. 2015 Oct;35(10):2100-6.

18 Leisser C, Hirnschall N, Palkovits S, Doeller B, Kefer K, Findl O. Intraoperative optical coherence tomography-guided membrane peeling for surgery of macular pucker: advantages and limitations. Ophthalmologica. 2019; 241(4):234-40.

19 Leisser C, Palkovits S, Hirnschall N, Ullrich $\mathrm{M}$, Hienert J, Zwickl H, et al. One-year results after internal limiting membrane flap transposition for surgical repair of macular holes with respect to microperimetry. Ophthalmic Res. 2019;61(2):83-7. 